\title{
MULTI-PEAK STANDING WAVES FOR NONLINEAR SCHRÖDINGER EQUATIONS WITH A GENERAL NONLINEARITY
}

\author{
JAEYOUNG BYEON \\ Department of Mathematics, \\ Pohang University of Science and Technology \\ Pohang, Kyungbuk 790-784, Republic of Korea \\ LOUIS JEANJEAN \\ Equipe de Mathématiques (UMR CNRS 6623), \\ Université de Franche-Comté, \\ 16 Route de Gray, 25030 Besançon, France
}

(Communicated by Aim Sciences)

\begin{abstract}
We consider singularly perturbed elliptic equations $\varepsilon^{2} \Delta u-V(x) u+$ $f(u)=0, x \in \mathbf{R}^{N}, N \geq 3$. For small $\varepsilon>0$, we glue together localized bound state solutions concentrating at isolated components of positive local minimum of $V$ under conditions on $f$ we believe to be almost optimal.
\end{abstract}

1. Introduction. This paper deals with the study of standing waves for the nonlinear Schrödinger equation

$$
i \hbar \frac{\partial \psi}{\partial t}+\frac{\hbar^{2}}{2} \Delta \psi-V(x) \psi+f(\psi)=0, \quad(t, x) \in \mathbf{R} \times \mathbf{R}^{N} .
$$

Here $\hbar$ denotes the Plank constant, $i$ the imaginary unit. For the physical background of this equation, we refer to the introduction in [7]. We assume that $f(\exp (i \theta) v)=\exp (i \theta) f(v)$ for $v \in \mathbf{R}$. A standing wave is a solution of the form $\psi(x, t)=\exp (-i E t / \hbar) v(x)$. Then, $\psi(x, t)$ is a solution of (1) if and only if the function $v$ satisfies

$$
\frac{\hbar^{2}}{2} \Delta v-(V(x)-E) v+f(v)=0 \quad \text { in } \quad \mathbf{R}^{N} .
$$

We are interested in positive solutions in $H^{1}\left(\mathbf{R}^{N}\right)$ for small $\hbar>0$. For small $\hbar>0$, these standing waves are referred as semi-classical states. For simplicity and without loss of generality, we write $V-E$ as $V$, i.e., we shift $E$ to 0 . Thus, we consider the following equation

$$
\varepsilon^{2} \Delta v-V(x) v+f(v)=0, \quad v>0, \quad v \in H^{1}\left(\mathbf{R}^{N}\right)
$$

when $\varepsilon>0$ is sufficiently small. Throughout the paper, the potential $V$ will be assumed to satisfy

(V1) $V \in C\left(\mathbf{R}^{N}, \mathbf{R}\right), 0 \leq V_{0} \equiv \inf _{\mathbf{R}^{N}} V(x)$ and $\liminf \inf _{|x| \rightarrow \infty} V(x)>0$.

2000 Mathematics Subject Classification. Primary: 35J20; Secondary: 35J60.

Key words and phrases. Variational methods, singularly perturbed elliptic problems. 
An interesting class of solutions of (3) are families of solutions which concentrate and develop spike layers, peaks, around certain points in $\mathbf{R}^{N}$ while vanishing elsewhere as $\varepsilon \rightarrow 0$. In the case $V_{0}>0$, the existence of single peak solutions was first studied by Floer and Weinstein [17]. For $N=1$ and $f(u)=u^{3}$, they construct a single peak solution concentrating around any given non-degenerate critical point of the potential $V(x)$. Oh [25] extended this result in higher dimension and for $f(u)=|u|^{p-1} u, 1<p<\frac{N+2}{N-2}$. The arguments in $[17,25]$ are based on a Lyapunov-

Schmidt reduction and rely on the uniqueness and non-degeneracy of the ground state solutions, namely of the positive least energy solutions, for the autonomous problems : for fixed $x_{0} \in \mathbf{R}^{N}$,

$$
\Delta v-V\left(x_{0}\right) v+f(v)=0 \quad \text { in } \quad \mathbf{R}^{N} \quad \text { and } \quad v \in H^{1}\left(\mathbf{R}^{N}\right) .
$$

These equations arise as limit equations corresponding to suitably rescaled solutions of (3). Subsequently reduction methods were also found suitable to find solutions of (3) concentrating around possibly degenerate, but structurally stable, critical points of $V(x)$, when the ground state solutions of the limit problems (4) are unique and non-degenerate. See, in particular, [1, 2, 11, 12, 22, 23] for reduction method approaches.

The uniqueness and non-degeneracy of the ground state solutions of (4) are, in general, difficult to check. They are known only for a rather restricted class of nonlinearities $f$. To attack the existence of positive solutions of (3) for more general nonlinearity, the variational approach, initiated by Rabinowitz [28], proved to be successful. In [28] he proves, by a mountain pass argument, the existence of positive solutions of (3) for small $\varepsilon>0$ whenever

$$
\liminf _{|x| \rightarrow \infty} V(x)>\inf _{x \in \mathbf{R}^{N}} V(x)>0 .
$$

These solutions concentrate around the global minimum points of $V$ when $\varepsilon \rightarrow 0$, as it was shown by $\mathrm{X}$. Wang [30]. This variational approach have been developed further by del Pino and Felmer, and some others. See, in particular, [7, 8, 13, 14, $15,16,19,21]$.

However, on one hand, in the classical paper [3], Berestycki and Lions showed the existence of least energy solutions for the limiting problem (4) with $V\left(x_{0}\right)>0$ when the nonlinearity $f$ satisfies almost necessary and sufficient conditions. On the other hand, in all previous mentioned works, even when they follow the variational approach, it is necessary to assume stronger conditions on $f$ that the ones of Berestycki and Lions. Very recently the authors in [5] manage to prove the existence of a solution of (3) concentrating around local minimum points of $V$ for small $\varepsilon>0$ only assuming these conditions. The approach in [5] is variational but quite different from the previous ones.

The main purpose of this paper is to develop the approach introduced in [5] as to be able to treat the existence of multi-peak solution of (3), exhibiting concentration at any prescribed set of local minima of the potential. After the initial work [26] this kind of solutions have been constructed in $[15,16,19]$ for some classes of nonlinearities. The conditions on $f$ depend on the kind of approach which is retained. In this paper we construct multi-peak solutions of (3) when the nonlinearity satisfies only the Berestycki-Lions's conditions. We believe our approach is also simpler than the previous ones. Finally in all the above mentioned works at the exception 
of $[6,7,8]$, it is assumed that $V_{0}>0$. Here we allow the possibility to have $V_{0}=0$. More precisely in addition to (V1) we assume on $V$.

(V2) There are bounded disjoint open sets $O^{1}, \cdots, O^{k}$ such that for $i=1, \cdots, k$,

$$
0<m_{i} \equiv \inf _{x \in O^{i}} V(x)<\min _{x \in \partial O^{i}} V(x) .
$$

For each $i \in\{1, \cdots, k\}$, we define

$$
M^{i} \equiv\left\{x \in O^{i} \mid V(x)=m_{i}\right\}
$$

and we set $Z \equiv\left\{x \in \mathbf{R}^{N} \mid V(x)=0\right\}$ and $m \equiv \min _{i \in\{1, \cdots, k\}} m_{i}$.

We also assume that $f: \mathbf{R}^{+} \rightarrow \mathbf{R}^{+}$is continuous and satisfies the following conditions.

(f1) $\lim _{t \rightarrow 0^{+}} f(t) / t=0$ if $Z=\emptyset$ and $\limsup _{t \rightarrow 0^{+}} \frac{f(t)}{t^{1+\mu}}<\infty$ for some $\mu>0$ if $Z \neq \emptyset$;

(f2) there exists some $p \in(1,(N+2) /(N-2)), N \geq 3$ such that $\lim \sup _{t \rightarrow \infty} f(t) / t^{p}$ $<\infty$

(f3) there exists $T>0$ such that $\frac{1}{2} m T^{2}<F(T)$, where $F(t)=\int_{0}^{t} f(s) d s$.

Theorem 1. Let $N \geq 3$. Suppose that (V1-2) and (f1-3) hold. Then for sufficiently small $\varepsilon>0$, there exists a positive solution $v_{\varepsilon}$ of (3) satisfying

(i) there exist $k$ local maximum points $x_{\varepsilon}^{i} \in O^{i}$ of $v_{\varepsilon}$ such that

$$
\lim _{\varepsilon \rightarrow 0} \max _{i=1, \cdots, k} \operatorname{dist}\left(x_{\varepsilon}^{i}, M^{i}\right)=0,
$$

and that $w_{\varepsilon}(x) \equiv v_{\varepsilon}\left(\varepsilon\left(x-x_{\varepsilon}^{i}\right)\right)$ converges (up to a subsequence) locally uniformly to a positive, least energy solution of

$$
\Delta u-m_{i} u+f(u)=0, \quad u>0, \quad u \in H^{1}\left(\mathbf{R}^{N}\right)
$$

(ii) for some $c, C>0$,

$$
v_{\varepsilon}(x) \leq C \exp \left(-\frac{c}{\varepsilon} \min _{i=1, \cdots, k}\left|x-x_{\varepsilon}^{i}\right|\right) .
$$

In [3] Berestycki and Lions proved that conditions (f2) and (f3) with $m=m_{i}$ are necessary for the existence of a non-trivial solution of the associated problem (5). In the case $Z \neq \emptyset$ we need an additional decay condition on $f$ at 0 , but when $Z=\emptyset$, the conditions (f1),(f2) and (f3) are the same then the Berestycki-Lions's conditions given in [3]. Thus, basically, the concentration phenomena occurs as soon as the $k$ equations (5) have a non-trivial solution.

The proof of Theorem 1 uses ideas introduced in [5], but is more involved. Defining $u(x)=v(\varepsilon x)$ and $V_{\varepsilon}(x)=V(\varepsilon x)$, equation (3) is equivalent to

$$
\Delta u-V_{\varepsilon} u+f(u)=0, \quad u>0, \quad u \in H^{1}\left(\mathbf{R}^{N}\right) .
$$

Roughly speaking we search directly a solution of (6) which consists essentially of $k$ disjoints parts, each part being close to a least energy solution of (5) associated to the corresponding $M^{i}$. Namely in our approach we take into account the shape and location of the solutions we expect to find. Thus on one hand we benefit from the advantage of the Lyapounov-Schmidt reduction approach, which is to discover the solution around a small neighborhood of a well chosen first approximation. On the other hand we do not need the uniqueness nor non-degeneracy of the least energy solutions of (5). Our approach is indeed purely variational. 
Finally we would like to mention that in [16] (see also [15]) existence of single and multi-peak solutions of (3) is obtained around any topologically non trivial critical point of $V$. This is at the expense of rather strong assumptions on $f$ and, as pointed out to us by M. del Pino and P. Felmer, it would be interesting to study if the approach of the present paper can be adapt to treat more general critical points of $V$.

2. Proof of Theorem 1. We shall find a solution of (3) working with (6). The variational framework is the following. Let $\widetilde{m}>0$ be a number such that

$$
\widetilde{m}<\min \left\{m, \liminf _{|x| \rightarrow \infty} V(x)\right\}
$$

and define $\tilde{V}_{\varepsilon}(x) \equiv \max \left\{\tilde{m}, V_{\varepsilon}(x)\right\}$. Let $H_{\varepsilon}$ be the completion of $C_{0}^{\infty}\left(\mathbf{R}^{N}\right)$ with respect to the norm

$$
\|u\|_{\varepsilon}=\left(\int_{\mathbf{R}^{N}}|\nabla u|^{2}+\tilde{V}_{\varepsilon} u^{2} d x\right)^{1 / 2} .
$$

We clearly have $H_{\varepsilon} \subset H^{1}\left(\mathbf{R}^{N}\right)$. From now on we define $M \equiv \cup_{i=1}^{k} M^{i}, O \equiv \cup_{i=1}^{k} O^{i}$ and for any set $B \subset \mathbf{R}^{N}$ and $\varepsilon, \alpha>0, B_{\varepsilon} \equiv\left\{x \in \mathbf{R}^{N} \mid \varepsilon x \in B\right\}$ and $B^{\delta} \equiv\{x \in$ $\left.\mathbf{R}^{N} \mid \operatorname{dist}(\mathrm{x}, \mathrm{B}) \leq \delta\right\}$. For $u \in H_{\varepsilon}$, let

$$
P_{\varepsilon}(u)=\frac{1}{2} \int_{\mathbf{R}^{N}}|\nabla u|^{2}+V_{\varepsilon} u^{2} d x-\int_{\mathbf{R}^{N}} F(u) d x
$$

(since we seek positive solutions, we assume without loss of generality that $f(t)=0$ for all $t \leq 0)$. Now, we define

$$
\chi_{\varepsilon}(x)=\left\{\begin{array}{ll}
0 & \text { if } x \in O_{\varepsilon} \\
\varepsilon^{-6 / \mu} & \text { if } x \notin O_{\varepsilon},
\end{array} \quad \chi_{\varepsilon}^{i}(x)= \begin{cases}0 & \text { if } x \in\left(O^{i}\right)_{\varepsilon} \\
\varepsilon^{-6 / \mu} & \text { if } x \notin\left(O^{i}\right)_{\varepsilon}\end{cases}\right.
$$

and

$$
Q_{\varepsilon}(u)=\left(\int_{\mathbf{R}^{N}} \chi_{\varepsilon} u^{2} d x-1\right)_{+}^{\frac{p+1}{2}}, \quad Q_{\varepsilon}^{i}(u)=\left(\int_{\mathbf{R}^{N}} \chi_{\varepsilon}^{i} u^{2} d x-1\right)_{+}^{\frac{p+1}{2}} .
$$

The functional $Q_{\varepsilon}$ will act as a penalization to force the concentration phenomena to occur inside $\mathrm{O}$. This type of penalization was first introduced in [8]. Finally we define the functionals $\Gamma_{\varepsilon}, \Gamma_{\varepsilon}^{1}, \cdots, \Gamma_{\varepsilon}^{k}: H_{\varepsilon} \rightarrow \mathbf{R}$ by

$$
\Gamma_{\varepsilon}(u)=P_{\varepsilon}(u)+Q_{\varepsilon}(u), \quad \Gamma_{\varepsilon}^{i}(u)=P_{\varepsilon}(u)+Q_{\varepsilon}^{i}(u), i=1, \cdots, k .
$$

It is standard to see that $\Gamma_{\varepsilon}, \Gamma_{\varepsilon}^{i} \in C^{1}\left(H_{\varepsilon}\right)$. Clearly a critical point of $P_{\varepsilon}$ corresponds to a solution of (6). To find solutions of (6) which concentrate in $O$ as $\varepsilon \rightarrow 0$, we shall search critical points of $\Gamma_{\varepsilon}$ for which $Q_{\varepsilon}$ is zero. First we study some properties of the solutions of $(5)$.

The following equations for $a>0$ are limiting equations of (6)

$$
\Delta u-a u+f(u)=0, \quad u>0, \quad u \in H^{1}\left(\mathbf{R}^{N}\right) .
$$

We define an energy functional for the problems (11) by

$$
L_{a}(u)=\frac{1}{2} \int_{\mathbf{R}^{N}}|\nabla u|^{2}+a u^{2} d x-\int_{\mathbf{R}^{N}} F(u) d x, \quad u \in H^{1}\left(\mathbf{R}^{N}\right) .
$$


In [3] Berestycki and Lions proved that, for any $a>0$, there exists a least energy solution of (11) if (f1),(f2) and (f3) with $m=a$ are satisfied and that each solution $U$ of (11) satisfies the Pohozaev's identity

$$
\frac{N-2}{2} \int_{\mathbf{R}^{N}}|\nabla U|^{2} d x+N \int_{\mathbf{R}^{N}} a \frac{u^{2}}{2}-F(u) d x=0 .
$$

From this we immediately deduce that, for any $U$ solution of (11)

$$
\frac{1}{N} \int_{\mathbf{R}^{N}}|\nabla U|^{2} d x=L_{a}(U) .
$$

Let $S_{a}$ be the set of least energy solutions $U$ of (11) satisfying $U(0)=\max _{x \in \mathbf{R}^{N}} U(x)$. Then, the following result was obtained in [5].

Proposition 1. For each $a>0$ and $N \geq 3, S_{a}$ is compact in $H^{1}\left(\mathbf{R}^{N}\right)$. Moreover, there exist $C, c>0$, independent of $U \in S_{a}$ such that

$$
U(x) \leq C \exp (-c|x|) .
$$

Let

$$
10 \delta \equiv \min \left\{\operatorname{dist}\left(M, \mathbf{R}^{N} \backslash O\right), \min _{i \neq j} \operatorname{dist}\left(O_{i}, O_{j}\right), \operatorname{dist}(O, Z)\right\} .
$$

We fix a $\beta \in(0, \delta)$ and a cutoff $\varphi \in C_{0}^{\infty}\left(\mathbf{R}^{N}\right)$ such that $0 \leq \varphi \leq 1, \varphi(x)=1$ for $|x| \leq \beta$ and $\varphi(x)=0$ for $|x| \geq 2 \beta$. Also, setting $\varphi_{\varepsilon}(y)=\varphi(\varepsilon y), y \in \mathbf{R}^{N}$, for each $x_{i} \in\left(M^{i}\right)^{\beta}$ and $U_{i} \in S_{m_{i}}$, we define

$$
U_{\varepsilon}^{x_{1}, \cdots, x_{k}}(y) \equiv \sum_{i=1}^{k} \varphi_{\varepsilon}\left(y-\frac{x_{i}}{\varepsilon}\right) U_{i}\left(y-\frac{x_{i}}{\varepsilon}\right) .
$$

We will find a solution, for sufficiently small $\varepsilon>0$, near the set

$$
X_{\varepsilon}=\left\{U_{\varepsilon}^{x_{1} \cdots, x_{k}}(y) \mid x_{i} \in\left(M^{i}\right)^{\beta} \text { and } U_{i} \in S_{m_{i}} \text { for each } i=1, \cdots, k\right\} .
$$

For each $i \in\{1, \cdots, k\}$ and $x_{i} \in M^{i}, U_{i} \in S_{m_{i}}$ arbitrary but fixed, we define

$$
W_{\varepsilon}^{i}(y)=\varphi_{\varepsilon}\left(y-\frac{x_{i}}{\varepsilon}\right) U_{i}\left(y-\frac{x_{i}}{\varepsilon}\right) .
$$

Setting $W_{\varepsilon, t}^{i}(y)=\varphi_{\varepsilon}\left(y-\frac{x_{i}}{\varepsilon}\right) U_{i}\left(\frac{y}{t}-\frac{x_{i}}{\varepsilon t}\right)$, we see that $\lim _{t \rightarrow 0}\left\|W_{\varepsilon, t}^{i}\right\|_{\varepsilon}=0$ and that $\Gamma_{\varepsilon}\left(W_{\varepsilon, t}^{i}\right)=P_{\varepsilon}\left(W_{\varepsilon, t}^{i}\right)$ for $t \geq 0$. Also, from (13) we see that for $U_{i, t}(x) \equiv U_{i}\left(\frac{x}{t}\right)$ we have

$$
\begin{aligned}
L_{m_{i}}\left(U_{i, t}\right) & =\int_{\mathbf{R}^{N}} \frac{t^{N-2}}{2}\left|\nabla U_{i}\right|^{2}+m_{i} \frac{t^{N}}{2} U_{i}^{2}-t^{N} F\left(U_{i}\right) d x \\
& =\left(\frac{t^{N-2}}{2}-\frac{(N-2) t^{N}}{2 N}\right) \int_{\mathbf{R}^{N}}\left|\nabla U_{i}\right|^{2} d x .
\end{aligned}
$$

Thus, there exists $T_{i}>0$ such that $L_{m_{i}}\left(U_{i, t}\right)<-2$ for $t \geq T_{i}$ and we can easily check that $\Gamma_{\varepsilon}\left(W_{\varepsilon, T_{i}}^{i}\right)<-2$ for any $\varepsilon>0$ sufficiently small.

Let $\gamma_{\varepsilon}^{i}(s)=W_{\varepsilon, s}^{i}(y)$ for $s>0$ and $\gamma_{\varepsilon}^{i}(0)=0$. For $s=\left(s_{1}, \cdots, s_{k}\right) \in T \equiv$ $\left[0, T_{1}\right] \times \cdot \times\left[0, T_{k}\right]$ we define

$$
\gamma_{\varepsilon}(s) \equiv \sum_{i=1}^{k} W_{\varepsilon, s_{i}}^{i} \quad \text { and } \quad D_{\varepsilon} \equiv \max _{s \in T} \Gamma_{\varepsilon}\left(\gamma_{\varepsilon}(s)\right) .
$$

Finally for each $i \in\{1, \cdots, k\}$, let $E_{i}=L_{m_{i}}(U)$ for $U \in S_{m_{i}}$. Then, for $E \equiv$ $\sum_{i=1}^{k} E_{i}$ we have 
Proposition 2. The followings hold

(i) $\lim _{\varepsilon \rightarrow 0} D_{\varepsilon}=E$,

(ii) $\lim \sup _{\varepsilon \rightarrow 0} \max _{s \in \partial T} \Gamma_{\varepsilon}\left(\gamma_{\varepsilon}(s)\right) \leq \tilde{E} \equiv \max \left\{E-E_{i} \mid i=1, \cdots, k\right\}<E$,

(iii) for each $d>0$, there exist $\alpha>0$ such that for sufficiently small $\varepsilon>0$,

$$
\Gamma_{\varepsilon}\left(\gamma_{\varepsilon}(s)\right) \geq C_{\varepsilon}-\alpha \text { implies that } \gamma_{\varepsilon}(s) \in X_{\varepsilon}^{d / 2} \text {. }
$$

Proof. Since $\operatorname{supp}\left(\gamma_{\varepsilon}(s)\right) \subset M_{\varepsilon}^{2 \beta}$ for each $s \in T$, it follows that $\Gamma_{\varepsilon}\left(\gamma_{\varepsilon}(s)\right)=$ $P_{\varepsilon}\left(\gamma_{\varepsilon}(s)\right)=\sum_{i=1}^{k} P_{\varepsilon}\left(\gamma_{\varepsilon}^{i}(s)\right)$. Now, for each $i \in\{1, \cdots, k\}$, we see from the decay property of $U_{i}$ and a change of variables that

$$
\begin{aligned}
P_{\varepsilon}\left(\gamma_{\varepsilon}^{i}(s)\right)= & \frac{1}{2} \int_{\mathbf{R}^{N}}\left|\nabla \gamma_{\varepsilon}^{i}(s)\right|^{2}+V_{\varepsilon}(x)\left(\gamma_{\varepsilon}^{i}(s)\right)^{2} d x-\int_{\mathbf{R}^{N}} F\left(\gamma_{\varepsilon}^{i}(s)\right) d x \\
= & \frac{1}{2} \int_{\mathbf{R}^{N}}\left|\nabla \gamma_{\varepsilon}^{i}(s)\right|^{2}+m_{i}\left(\gamma_{\varepsilon}^{i}(s)\right)^{2} d x-\int_{\mathbf{R}^{N}} F\left(\gamma_{\varepsilon}^{i}(s)\right) d x \\
& +\frac{1}{2} \int_{\mathbf{R}^{N}}\left(V_{\varepsilon}(x)-m_{i}\right)\left(\gamma_{\varepsilon}^{i}(s)\right)^{2} d x \\
= & \frac{s^{N-2}}{2} \int_{\mathbf{R}^{N}}\left|\nabla U_{i}\right|^{2} d x+s^{N} \int_{\mathbf{R}^{N}} \frac{1}{2} m_{i} U_{i}^{2}-F\left(U_{i}\right) d x+O(\varepsilon) .
\end{aligned}
$$

Then, from the Pohozaev identity (13), we see that

$$
P_{\varepsilon}\left(\gamma_{\varepsilon}^{i}(s)\right)=\left(\frac{s^{N-2}}{2}-\frac{N-2}{2 N} s^{N}\right) \int_{\mathbf{R}^{N}}\left|\nabla U_{i}\right|^{2} d x+O(\varepsilon) .
$$

Also

$$
\max _{t \in(0, \infty)}\left(\frac{t^{N-2}}{2}-\frac{N-2}{2 N} t^{N}\right) \int_{\mathbf{R}^{N}}\left|\nabla U_{i}\right|^{2} d x=E_{i} .
$$

At this point we deduce that (i) and (ii) hold. To conclude we just observe that for $g(t)=\frac{t^{N-2}}{2}-\frac{N-2}{2 N} t^{N}$,

$$
g^{\prime}(t) \begin{cases}>0 & \text { for } t \in(0,1) \\ =0 & \text { for } t=1 \\ <0 & \text { for } t>1\end{cases}
$$

and $g^{\prime \prime}(1)=2-N<0$.

Now let

$$
\Phi_{\varepsilon}^{i}=\left\{\varphi \in C\left(\left[0, T_{i}\right], H_{\varepsilon}\right) \mid \varphi\left(s_{i}\right)=\gamma_{\varepsilon}^{i}\left(s_{i}\right) \text { for } s_{i}=0 \text { or } T_{i}\right\}
$$

and

$$
C_{\varepsilon}^{i}=\inf _{\varphi \in \Phi_{\varepsilon}^{i}} \max _{s_{i} \in\left[0, T_{i}\right]} \Gamma_{\varepsilon}^{i}\left(\varphi\left(s_{i}\right)\right) .
$$

For future reference we need the following estimate.

\section{Proposition 3.}

$$
\liminf _{\varepsilon \rightarrow 0} C_{\varepsilon}^{i} \geq E_{i}, \quad i=1, \cdots, k .
$$

Proof. The proof is identical to the one of Proposition 3 in [5] where we observe

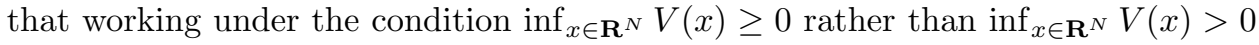
is sufficient.

Now we define

$$
\Gamma_{\varepsilon}^{\alpha}=\left\{u \in H_{\varepsilon} \mid \Gamma_{\varepsilon}(u) \leq \alpha\right\}
$$


and for a set $A \subset H_{\varepsilon}$ and $\alpha>0$, let $A^{\alpha} \equiv\left\{u \in H_{\varepsilon} \mid\|u-A\| \leq \alpha\right\}$.

Proposition 4. Let $\left\{\varepsilon_{j}\right\}_{j=1}^{\infty}$ be such that $\lim _{j \rightarrow \infty} \varepsilon_{j}=0$ and $\left\{u_{\varepsilon_{j}}\right\} \in X_{\varepsilon_{j}}^{d}$ such that

$$
\lim _{j \rightarrow \infty} \Gamma_{\varepsilon_{j}}\left(u_{\varepsilon_{j}}\right) \leq E \text { and } \lim _{j \rightarrow \infty} \Gamma_{\varepsilon_{j}}^{\prime}\left(u_{\varepsilon_{j}}\right)=0 .
$$

Then, for sufficiently small $d>0$, there exist, up to a subsequence, $\left\{y_{j}^{i}\right\}_{j=1}^{\infty} \subset \mathbf{R}^{N}$, $i=1, \cdots, k, x^{i} \in M^{i}, U_{i} \in S_{m_{i}}$ such that

$$
\lim _{j \rightarrow \infty}\left|\varepsilon_{j} y_{j}^{i}-x^{i}\right|=0 \text { and } \lim _{j \rightarrow \infty}\left\|u_{\varepsilon_{j}}-\sum_{i=1}^{k} \varphi_{\varepsilon_{j}}\left(\cdot-y_{j}^{i}\right) U_{i}\left(\cdot-y_{j}^{i}\right)\right\|_{\varepsilon_{j}}=0 .
$$

Proof. For the sake of convenience, we write $\varepsilon$ for $\varepsilon_{j}$. From Proposition 1, we know that the $S_{m_{i}}$ are compact. Then there exist $Z_{i} \in S_{m_{i}}$ and $x \in\left(M^{i}\right)^{\beta}$ for $i=1, \cdots, k$, such that, passing to a subsequence still denoted $\left\{u_{\varepsilon}\right\}$,

$$
\left\|u_{\varepsilon}-\sum_{i=1}^{k} \varphi_{\varepsilon}\left(\cdot-x^{i} / \varepsilon\right) Z_{i}\left(\cdot-x^{i} / \varepsilon\right)\right\|_{\varepsilon} \leq 2 d
$$

for small $\varepsilon>0$. We denote $u_{\varepsilon}^{1}=\sum_{i=1}^{k} \varphi_{\varepsilon}\left(\cdot-x^{i} / \varepsilon\right) u_{\varepsilon}$ and $u_{\varepsilon}^{2}=u_{\varepsilon}-u_{\varepsilon}^{1}$. As a first step in the proof of the Proposition we shall prove that

$$
\Gamma_{\varepsilon}\left(u_{\varepsilon}\right) \geq \Gamma_{\varepsilon}\left(u_{\varepsilon}^{1}\right)+\Gamma_{\varepsilon}\left(u_{\varepsilon}^{2}\right)+O(\varepsilon) .
$$

Suppose there exist $x_{\varepsilon} \in \cup_{i=1}^{k} B\left(x^{i} / \varepsilon, 2 \beta / \varepsilon\right) \backslash B\left(x^{i} / \varepsilon, \beta / \varepsilon\right)$ and $R>0$ satisfying $\liminf _{\varepsilon \rightarrow 0} \int_{B\left(x_{\varepsilon}, R\right)}\left(u_{\varepsilon}\right)^{2} d y>0$. Taking a subsequence, we can assume that $\varepsilon x_{\varepsilon} \rightarrow x_{0}$ with $x_{0}$ in the closure of $\cup_{i=1}^{k} B\left(x^{i}, 2 \beta\right) \backslash B\left(x^{i}, \beta\right)$ and that $u_{\varepsilon}\left(\cdot+x_{\varepsilon}\right) \rightarrow \tilde{W} \neq 0$ weakly in $H^{1}\left(\mathbf{R}^{N}\right)$ for some $\tilde{W} \in H^{1}\left(\mathbf{R}^{N}\right)$. Moreover $\tilde{W}$ satisfies

$$
\Delta \tilde{W}(y)-V\left(x_{0}\right) \tilde{W}(y)+f(\tilde{W}(y))=0 \text { for } y \in \mathbf{R}^{N} .
$$

By definition, $L_{V\left(x_{0}\right)}(\tilde{W}) \geq E_{V\left(x_{0}\right)}$. Also, for large $R>0$

$$
\liminf _{\varepsilon \rightarrow 0} \int_{B\left(x_{\varepsilon}, R\right)}\left|\nabla u_{\varepsilon}\right|^{2} d y \geq \frac{1}{2} \int_{\mathbf{R}^{N}}|\nabla \tilde{W}|^{2} d y .
$$

Now, recalling from [20] that $E_{a}>E_{b}$ if $a>b$, we see that $E_{V\left(x_{0}\right)} \geq E_{m}$, since $V\left(x_{0}\right) \geq m$. Thus, from (14) and (18) we get that

$$
\liminf _{\varepsilon \rightarrow 0} \int_{B\left(x_{\varepsilon}, R\right)}\left|\nabla u_{\varepsilon}\right|^{2} d y \geq \frac{N}{2} L_{V\left(x_{0}\right)}(\tilde{W}) \geq \frac{N}{2} E_{m}>0 .
$$

Then, taking $d>0$ sufficiently small, we get a contradiction with (16). Since there does not exist such a sequence $\left\{x_{\varepsilon}\right\}_{\varepsilon}$ we deduce from a result of P.L. Lions (see [24, Lemma I.1]) that

$$
\liminf _{\varepsilon \rightarrow 0} \int_{\cup_{i=1}^{k} B\left(x^{i} / \varepsilon, 2 \beta / \varepsilon\right) \backslash B\left(x^{i} / \varepsilon, \beta / \varepsilon\right)}\left|u_{\varepsilon}\right|^{p+1} d y=0 .
$$

Thus, we can derive using (f1),(f2) and the boundedness of $\left\{\left\|u_{\varepsilon}\right\|_{L^{2}\left(\cup_{i=1}^{k} B\left(x^{i} / \varepsilon, 2 \beta / \varepsilon\right)\right)}\right\}_{\varepsilon}$ that

$$
\lim _{\varepsilon \rightarrow 0} \int_{\mathbf{R}^{N}} F\left(u_{\varepsilon}\right)-F\left(u_{\varepsilon}^{1}\right)-F\left(u_{\varepsilon}^{2}\right) d y=0 .
$$


At this point, writing

$$
\begin{aligned}
\Gamma_{\varepsilon}\left(u_{\varepsilon}\right) & =\Gamma_{\varepsilon}\left(u_{\varepsilon}^{1}\right)+\Gamma_{\varepsilon}\left(u_{\varepsilon}^{2}\right) \\
& +\int_{\mathbf{R}^{N}} \varphi_{\varepsilon}\left(1-\varphi_{\varepsilon}\right)\left|\nabla u_{\varepsilon}\right|^{2}+V_{\varepsilon} \varphi_{\varepsilon}\left(1-\varphi_{\varepsilon}\right) u_{\varepsilon}^{2} d y \\
& -\int_{\mathbf{R}^{N}} F\left(u_{\varepsilon}\right)-F\left(u_{\varepsilon}^{1}\right)-F\left(u_{\varepsilon}^{2}\right) d y+O(\varepsilon),
\end{aligned}
$$

the inequality (17) follows.

We now estimate $\Gamma_{\varepsilon}\left(u_{\varepsilon}^{2}\right)$. We have

$$
\begin{aligned}
\Gamma_{\varepsilon}\left(u_{\varepsilon}^{2}\right) \geq P_{\varepsilon}\left(u_{\varepsilon}^{2}\right) & =\frac{1}{2} \int_{\mathbf{R}^{N}}\left|\nabla u_{\varepsilon}^{2}\right|^{2}+\tilde{V}_{\varepsilon}\left|u_{\varepsilon}^{2}\right|^{2} d x-\frac{1}{2} \int_{\mathbf{R}^{N}}\left(\tilde{V}_{\varepsilon}-V_{\varepsilon}\right)\left|u_{\varepsilon}^{2}\right|^{2} d x \\
& -\int_{\mathbf{R}^{N}} F\left(u_{\varepsilon}^{2}\right) d x \\
& \geq \frac{1}{2}\left\|u_{\varepsilon}^{2}\right\|_{\varepsilon}^{2}-\frac{\tilde{m}}{2} \int_{\mathbf{R}^{N} \backslash O_{\varepsilon}}\left|u_{\varepsilon}^{2}\right|^{2} d x-\int_{\mathbf{R}^{N}} F\left(u_{\varepsilon}^{2}\right) d x .
\end{aligned}
$$

Here we have use the fact that $\tilde{V}_{\varepsilon}-V_{\varepsilon}=0$ on $O_{\varepsilon}$ and $\left|\tilde{V}_{\varepsilon}-V_{\varepsilon}\right| \leq \tilde{m}$ on $\mathbf{R}^{N} \backslash O_{\varepsilon}$. Because of (f1),(f2) for some $C_{1}, C_{2}>0$,

$$
\begin{aligned}
\int_{\mathbf{R}^{N}} F\left(u_{\varepsilon}^{2}\right) d x & \leq \frac{\tilde{m}}{4} \int_{\mathbf{R}^{N}}\left(u_{\varepsilon}^{2}\right)^{2} d x+C_{1} \int_{\mathbf{R}^{N}}\left(u_{\varepsilon}^{2}\right)^{\frac{2 N}{N-2}} d x \\
& \leq \frac{\tilde{m}}{4} \int_{\mathbf{R}^{N}}\left(u_{\varepsilon}^{2}\right)^{2} d x+C_{2}\left\|u_{\varepsilon}^{2}\right\|_{\varepsilon}^{\frac{2 N}{N-2}} .
\end{aligned}
$$

Since $\left\{u_{\varepsilon}\right\}_{\varepsilon}$ is bounded, we see from (16) that $\left\|u_{\varepsilon}^{2}\right\|_{\varepsilon} \leq 4 d$ for small $\varepsilon>0$. Thus taking $d>0$ small enough we have

$$
\frac{1}{2}\left\|u_{\varepsilon}^{2}\right\|_{\varepsilon}^{2}-\int_{\mathbf{R}^{N}} F\left(u_{\varepsilon}^{2}\right) d x \geq\left\|u_{\varepsilon}^{2}\right\|_{\varepsilon}^{2}\left(\frac{1}{4}-C_{2}(4 d)^{4 /(N-2)}\right) \geq 0 .
$$

Now note that $P_{\varepsilon}$ is uniformly bounded in $X_{\varepsilon}^{d}$ for small $\varepsilon>0$. Thus, so is $Q_{\varepsilon}$. This implies that for some $C>0$,

$$
\int_{\mathbf{R}^{N} \backslash O_{\varepsilon}}\left(u_{\varepsilon}^{2}\right)^{2} d x \leq C \varepsilon^{6 / \mu}
$$

and recording $(20),(21)$ we deduce that $\Gamma_{\varepsilon}\left(u_{\varepsilon}^{2}\right) \geq O(\varepsilon)$. For future reference note also that denoting $p+1=2 s+(1-s) \frac{2 N}{N-2}, s \in(0,1)$, we see from (f1), (f2), (22) and using the interpolation and Sobolev inequalities, that for some $C_{1}, C_{2}>0$,

$$
\begin{aligned}
\int_{\mathbf{R}^{N} \backslash O_{\varepsilon}} F\left(u_{\varepsilon}^{2}\right) d x & \leq C_{1} \int_{\mathbf{R}^{N} \backslash O_{\varepsilon}}\left(u_{\varepsilon}^{2}\right)^{2}+\left(u_{\varepsilon}^{2}\right)^{p+1} d x \\
& \leq C_{1} \int_{\mathbf{R}^{N} \backslash O_{\varepsilon}}\left(u_{\varepsilon}\right)^{2} d x \\
& +C_{2}\left(\int_{\mathbf{R}^{N} \backslash O_{\varepsilon}}\left(u_{\varepsilon}\right)^{2} d x\right)^{s}\left\|u_{\varepsilon}\right\|_{\varepsilon}^{(1-s) \frac{2 N}{N-2}}
\end{aligned}
$$

Thus

$$
\lim _{\varepsilon \rightarrow 0} \int_{\mathbf{R}^{N} \backslash O_{\varepsilon}} F\left(u_{\varepsilon}^{2}\right) d x=0
$$

Now for $i=1, \cdots, k$, we define $u_{\varepsilon}^{1, i}(x)=u_{\varepsilon}^{1}(x)$ for $x \in O_{\varepsilon}^{i}, u_{\varepsilon}^{1, i}(x)=0$ for $x \notin O_{\varepsilon}^{i}$. Also we set $W_{\varepsilon}^{i}(y)=u_{\varepsilon}^{1, i}\left(y+x^{i} / \varepsilon\right)$. Now we fix an arbitrary $i \in\{1, \cdots, k\}$. Taking 
a subsequence we can assume that, $W_{\varepsilon}^{i} \rightarrow W_{i}$ weakly in $H^{1}\left(\mathbf{R}^{N}\right)$ for some $W_{i} \in$ $H^{1}\left(\mathbf{R}^{N}\right)$. Moreover $W_{i}$ satisfies

$$
\Delta W_{i}(y)-V\left(x^{i}\right) W_{i}(y)+f\left(W_{i}(y)\right)=0 \text { for } y \in \mathbf{R}^{N} .
$$

From the maximum principle we see that $W_{i}$ is positive. Let us prove that $W_{\varepsilon}^{i} \rightarrow W_{i}$ strongly in $H^{1}\left(\mathbf{R}^{N}\right)$. Suppose there exist $R>0$ and a sequence $\left\{z_{\varepsilon}\right\}_{\varepsilon}$ with $z_{\varepsilon} \in B\left(x^{i} / \varepsilon, 2 \beta / \varepsilon\right)$ satisfying

$$
\liminf _{\varepsilon \rightarrow 0}\left|z_{\varepsilon}-x^{i} / \varepsilon\right|=\infty \text { and } \quad \liminf _{\varepsilon \rightarrow 0} \int_{B\left(z_{\varepsilon}, R\right)}\left(u_{\varepsilon}^{1, i}\right)^{2} d y>0 .
$$

We may assume that $\varepsilon z_{\varepsilon} \rightarrow c_{i} \in O^{i}$ as $\varepsilon \rightarrow 0$. Then, $\tilde{W}_{\varepsilon}^{i}(y)=u_{\varepsilon}^{1, i}\left(y+z_{\varepsilon}\right)$ converges weakly to $\tilde{W}_{i}$ in $H^{1}\left(\mathbf{R}^{N}\right)$ satisfying

$$
\Delta \tilde{W}_{i}-V\left(c_{i}\right) \tilde{W}_{i}+f\left(\tilde{W}_{i}\right)=0, \text { for } y \in \mathbf{R}^{N} .
$$

At this point as before we get a contradiction and then using (f1),(f2) and [24, Lemma I.1] it follows that

$$
\lim _{\varepsilon \rightarrow 0} \int_{\mathbf{R}^{N}} F\left(W_{\varepsilon}^{i}\right) d x \rightarrow \int_{\mathbf{R}^{N}} F\left(W_{i}\right) d x
$$

Then from the weak convergence of $W_{\varepsilon}^{i}$ to $W_{i}$ in $H^{1}\left(\mathbf{R}^{N}\right)$ we get, for any $i \in$ $\{1, \cdots, k\}$

$$
\begin{aligned}
& \limsup _{\varepsilon \rightarrow 0} \Gamma_{\varepsilon}\left(u_{\varepsilon}^{1, i}\right) \\
\geq & \liminf _{\varepsilon \rightarrow 0} P_{\varepsilon}\left(u_{\varepsilon}^{1, i}\right) \\
= & \liminf _{\varepsilon \rightarrow 0} \frac{1}{2} \int_{\mathbf{R}^{N}}\left|\nabla W_{\varepsilon}^{i}(y)\right|^{2}+V\left(\varepsilon y+x^{i}\right)\left(W_{\varepsilon}^{i}\right)^{2}(y) d y-\int_{\mathbf{R}^{N}} F\left(W_{\varepsilon}^{i}(y)\right) d y \\
\geq & \frac{1}{2} \int_{\mathbf{R}^{N}}\left|\nabla W_{i}\right|^{2}+V\left(x^{i}\right)\left(W_{i}\right)^{2} d y-\int_{\mathbf{R}^{N}} F\left(W_{i}\right) d y \geq E_{i} .
\end{aligned}
$$

Now by (17),

$$
\begin{aligned}
\limsup _{\varepsilon \rightarrow 0}\left(\Gamma_{\varepsilon}\left(u_{\varepsilon}^{2}\right)+\sum_{i=1}^{k} \Gamma_{\varepsilon}\left(u_{\varepsilon}^{1, i}\right)\right) & =\limsup _{\varepsilon \rightarrow 0}\left(\Gamma_{\varepsilon}\left(u_{\varepsilon}^{2}\right)+\Gamma_{\varepsilon}\left(u_{\varepsilon}^{1}\right)\right) \\
& \leq \limsup _{\varepsilon \rightarrow 0} \Gamma_{\varepsilon}\left(u_{\varepsilon}\right) \leq E=\sum_{i=1}^{k} E_{i} .
\end{aligned}
$$

Thus, since $\Gamma_{\varepsilon}\left(u_{\varepsilon}^{2}\right) \geq O(\varepsilon)$ we deduce from (26), (27) that, for any $i \in\{1, \cdots k\}$

$$
\lim _{\varepsilon \rightarrow 0} \Gamma_{\varepsilon}\left(u_{\varepsilon}^{1, i}\right)=E_{i}
$$

Now (26), (28) implies that $L_{V\left(x^{i}\right)}\left(W_{i}\right)=E_{i}$ and from [20], we see that $x^{i} \in M^{i}$. At this point it is clear that $W_{i}(y)=U_{i}\left(y-z_{i}\right)$ with $U_{i} \in S_{m_{i}}$ and $z_{i} \in \mathbf{R}^{N}$. Finally, using (25), (28) and the fact that $V \geq V\left(x^{i}\right)$ on $O^{i}$, we get from (26) that

$$
\begin{aligned}
\int_{\mathbf{R}^{N}}\left|\nabla W_{i}\right|^{2}+V\left(x^{i}\right) W_{i}^{2} d y & \geq \limsup _{\varepsilon \rightarrow 0} \int_{\mathbf{R}^{N}}\left|\nabla u_{\varepsilon}^{1, i}(y)\right|^{2}+V(\varepsilon y)\left(u_{\varepsilon}^{1, i}\right)^{2}(y) d y \\
& \geq \limsup _{\varepsilon \rightarrow 0} \int_{\mathbf{R}^{N}}\left|\nabla u_{\varepsilon}^{1, i}(y)\right|^{2}+V\left(x^{i}\right)\left(u_{\varepsilon}^{1, i}\right)^{2}(y) d y \\
& \geq \limsup _{\varepsilon \rightarrow 0} \int_{\mathbf{R}^{N}}\left|\nabla W_{\varepsilon}^{i}(y)\right|^{2}+V\left(x^{i}\right)\left(W_{\varepsilon}^{i}(y)\right)^{2} d y .
\end{aligned}
$$


This proves the strong convergence of $W_{\varepsilon}^{i}$ to $W_{i}$ in $H^{1}\left(\mathbf{R}^{N}\right)$. In particular setting $y_{\varepsilon}^{i}=x^{i} / \varepsilon+z_{i}$ we have $u_{\varepsilon}^{1, i} \rightarrow \varphi_{\varepsilon}\left(\cdot-y_{\varepsilon}^{i}\right) U_{i}\left(\cdot-y_{\varepsilon}^{i}\right)$ strongly in $H^{1}\left(\mathbf{R}^{N}\right)$. This means that $u_{\varepsilon}^{1, i} \rightarrow \varphi_{\varepsilon}\left(\cdot-y_{\varepsilon}^{i}\right) U_{i}\left(\cdot-y_{\varepsilon}^{i}\right)$ strongly in $H_{\varepsilon}$ and thus

$$
u_{\varepsilon}^{1}=\sum_{i=1}^{k} u_{\varepsilon}^{1, i} \rightarrow \sum_{i=1}^{k} \varphi_{\varepsilon}\left(\cdot-y_{\varepsilon}^{i}\right) U_{i}\left(\cdot-y_{\varepsilon}^{i}\right)
$$

strongly in $H_{\varepsilon}$. To conclude the proof of the Proposition, it suffices to show that $u_{\varepsilon}^{2} \rightarrow 0$ in $H_{\varepsilon}$. Since $E \geq \lim _{\varepsilon \rightarrow 0} \Gamma_{\varepsilon}\left(u_{\varepsilon}\right)$ and $\lim _{\varepsilon \rightarrow 0} \Gamma_{\varepsilon}\left(u_{\varepsilon}^{1}\right)=E$ we deduce, using (17) that $\lim _{\varepsilon \rightarrow 0} \Gamma_{\varepsilon}\left(u_{\varepsilon}^{2}\right)=0$. Now from (20), (21), (24) we get that $u_{\varepsilon}^{2} \rightarrow 0$ in $H_{\varepsilon}$ and this completes the proof.

Proposition 5. For sufficiently small $d_{1}>d_{2}>0$, there exist constants $\omega>0$ and $\varepsilon_{0}>0$ such that $\left|\Gamma_{\varepsilon}^{\prime}(u)\right| \geq \omega$ for $u \in \Gamma_{\varepsilon}^{D_{\varepsilon}} \cap\left(X_{\varepsilon}^{d_{1}} \backslash X_{\varepsilon}^{d_{2}}\right)$ and $\varepsilon \in\left(0, \varepsilon_{0}\right)$.

Proof. To the contrary, suppose that for small $d_{1}>d_{2}>0$, there exist $\left\{\varepsilon_{j}\right\}_{j=1}^{\infty}$ with $\lim _{j \rightarrow \infty} \varepsilon_{j}=0$ and $u_{\varepsilon_{j}} \in X_{\varepsilon_{j}}^{d_{1}} \backslash X_{\varepsilon_{j}}^{d_{2}}$ satisfying $\lim _{j \rightarrow \infty} \Gamma_{\varepsilon_{j}}\left(u_{\varepsilon_{j}}\right) \leq E$ and $\lim _{j \rightarrow \infty} \Gamma_{\varepsilon_{j}}^{\prime}\left(u_{\varepsilon_{j}}\right)=0$. For the sake of convenience, we write $\varepsilon$ for $\varepsilon_{j}$. By Proposition 4, there exists $\left\{y_{\varepsilon}^{i}\right\}_{\varepsilon} \subset \mathbf{R}^{N}, i=1, \cdots, k, x^{i} \in M^{i}, U_{i} \in S_{m_{i}}$ such that

$$
\lim _{\varepsilon \rightarrow 0}\left|\varepsilon y_{\varepsilon}^{i}-x^{i}\right|=0 \quad \text { and } \quad \lim _{\varepsilon \rightarrow 0}\left\|u_{\varepsilon}-\sum_{i=1}^{k} \varphi_{\varepsilon}\left(\cdot-y_{\varepsilon}^{i}\right) U_{i}\left(\cdot-y_{\varepsilon}^{i}\right)\right\|_{\varepsilon}=0 .
$$

By the definition of $X_{\varepsilon}$, we see that $\lim _{\varepsilon \rightarrow 0} \operatorname{dist}\left(u_{\varepsilon}, X_{\varepsilon}\right)=0$. This contradicts that $u_{\varepsilon} \notin X_{\varepsilon}^{d_{2}}$, and completes the proof.

Following Proposition 5 we fix a $d>0$ and corresponding $\omega>0$ and $\varepsilon_{0}>0$ such that $\left|\Gamma_{\varepsilon}^{\prime}(u)\right| \geq \omega$ for $u \in \Gamma_{\varepsilon}^{D_{\varepsilon}} \cap\left(X_{\varepsilon}^{d} \backslash X_{\varepsilon}^{d / 2}\right)$ and $\varepsilon \in\left(0, \varepsilon_{0}\right)$. Now, we obtain the following proposition.

Proposition 6. For sufficiently small fixed $\varepsilon>0$, there exists a sequence $\left\{u_{n}\right\}_{n=1}^{\infty}$ $\subset X_{\varepsilon}^{d} \cap \Gamma_{\varepsilon}^{D_{\varepsilon}}$ such that $\Gamma_{\varepsilon}^{\prime}\left(u_{n}\right) \rightarrow 0$ as $n \rightarrow \infty$.

Proof. By Proposition 2 (iii), there exists $\alpha \in(0, E-\tilde{E})$ such that for sufficiently small $\varepsilon>0$,

$$
\Gamma_{\varepsilon}\left(\gamma_{\varepsilon}(s)\right) \geq D_{\varepsilon}-\alpha \text { implies that } \gamma_{\varepsilon}(s) \in X_{\varepsilon}^{d / 2} .
$$

If Proposition 6 does not hold for sufficiently small $\varepsilon>0$, there exists $a(\varepsilon)>0$ such that $\left|\Gamma_{\varepsilon}^{\prime}(u)\right| \geq a(\varepsilon)$ on $X_{\varepsilon}^{d} \cap \Gamma_{\varepsilon}^{D_{\varepsilon}}$. Note from Proposition 5 that there exists $\omega>0$, independent of $\varepsilon>0$, such that $\left|\Gamma_{\varepsilon}^{\prime}(u)\right| \geq \omega$ for $u \in \Gamma_{\varepsilon}^{D_{\varepsilon}} \cap\left(X_{\varepsilon}^{d} \backslash X_{\varepsilon}^{d / 2}\right)$. Thus, by a deformation argument, for sufficiently small $\varepsilon>0$ there exists a $\mu \in(0, \alpha)$ and a path $\gamma \in \Phi_{\varepsilon}$ satisfying

$$
\begin{gathered}
\gamma(s)=\gamma_{\varepsilon}(s) \quad \text { for } \gamma_{\varepsilon}(s) \in \Gamma_{\varepsilon}^{D_{\varepsilon}-\alpha} \\
\gamma(s) \in X_{\varepsilon}^{d} \quad \text { for } \gamma_{\varepsilon}(s) \notin \Gamma_{\varepsilon}^{D_{\varepsilon}-\alpha}
\end{gathered}
$$

and

$$
\Gamma_{\varepsilon}(\gamma(s))<D_{\varepsilon}-\mu, \quad s \in T .
$$

Let $\psi \in C_{0}^{\infty}\left(\mathbf{R}^{N}\right)$ be such that $\psi(x)=1$ for $x \in O^{\delta}, \psi(x)=0$ for $x \notin O^{2 \delta}$, $\psi(x) \in[0,1]$ and $|\nabla \psi| \leq 2 / \delta$. For $\gamma(s) \in X_{\varepsilon}^{d}$, we define $\gamma^{1}(s)=\psi_{\varepsilon} \gamma(s)$ and 
$\gamma^{2}(s)=\left(1-\psi_{\varepsilon}\right) \gamma(s)$ where $\psi_{\varepsilon}(x)=\psi(\varepsilon x)$. Note that

$$
\begin{aligned}
\Gamma_{\varepsilon}(\gamma(s))=\quad & \Gamma_{\varepsilon}\left(\gamma^{1}(s)\right)+\Gamma_{\varepsilon}\left(\gamma^{2}(s)\right) \\
& +\int_{\mathbf{R}^{N}} \psi_{\varepsilon}\left(1-\psi_{\varepsilon}\right)|\nabla \gamma(s)|^{2}+V_{\varepsilon} \psi_{\varepsilon}\left(1-\psi_{\varepsilon}\right)(\gamma(s))^{2} d x \\
& +Q_{\varepsilon}(\gamma(s))-Q_{\varepsilon}\left(\gamma^{1}(s)\right)-Q_{\varepsilon}\left(\gamma^{2}(s)\right) \\
& -\int_{\mathbf{R}^{N}} F(\gamma(s))-F\left(\gamma^{1}(s)\right)-F\left(\gamma^{2}(s)\right) d x+O(\varepsilon) .
\end{aligned}
$$

Since for $A, B \geq 0,(A+B-1)_{+} \geq(A-1)_{+}+(B-1)_{+}$and since $p+1 \geq 2$ it follows that

$$
\begin{aligned}
Q_{\varepsilon}(\gamma(s)) & =\left(\int_{\mathbf{R}^{N}} \chi_{\varepsilon}\left(\gamma^{1}(s)+\gamma^{2}(s)\right)^{2} d x-1\right)_{+}^{\frac{p+1}{2}} \\
& \geq\left(\int_{\mathbf{R}^{N}} \chi_{\varepsilon}\left(\gamma^{1}(s)\right)^{2} d x+\int_{\mathbf{R}^{N}} \chi_{\varepsilon}\left(\gamma^{2}(s)\right)^{2} d x-1\right)_{+}^{\frac{p+1}{2}} \\
& \geq\left(\int_{\mathbf{R}^{N}} \chi_{\varepsilon}\left(\gamma^{1}(s)\right)^{2} d x-1\right)_{+}^{\frac{p+1}{2}}+\left(\int_{\mathbf{R}^{N}} \chi_{\varepsilon}\left(\gamma^{2}(s)\right)^{2} d x-1\right)_{+}^{\frac{p+1}{2}} \\
& =Q_{\varepsilon}\left(\gamma^{1}(s)\right)+Q_{\varepsilon}\left(\gamma^{2}(s)\right) .
\end{aligned}
$$

Also, using (22), we see reasoning as in (23), that

$$
\begin{aligned}
& \int_{\mathbf{R}^{N}}\left|F(\gamma(s))-F\left(\gamma^{1}(s)\right)-F\left(\gamma^{2}(s)\right)\right| d x \\
& =\int_{O_{\varepsilon}^{2 \delta} \backslash O_{\varepsilon}^{\delta}}\left|F(\gamma(s))-F\left(\gamma^{1}(s)\right)-F\left(\gamma^{2}(s)\right)\right| d x=O(\varepsilon) .
\end{aligned}
$$

Thus, we see that

$$
\Gamma_{\varepsilon}(\gamma(s)) \geq \Gamma_{\varepsilon}\left(\gamma^{1}(s)\right)+\Gamma_{\varepsilon}\left(\gamma^{2}(s)\right)+O(\varepsilon) .
$$

Also

$$
\Gamma_{\varepsilon}\left(\gamma^{2}(s)\right) \geq-\int_{\mathbf{R}^{N} \backslash O_{\varepsilon}} F\left(\gamma^{2}(s)\right) d x
$$

and again from (22), as in (23), we see that $\Gamma_{\varepsilon}\left(\gamma^{2}(s)\right) \geq O(\varepsilon)$. Therefore it follows that

$$
\Gamma_{\varepsilon}(\gamma(s)) \geq \Gamma_{\varepsilon}\left(\gamma^{1}(s)\right)+O(\varepsilon) .
$$

For $i=1, \cdots, k$, we define $\gamma^{1, i}(s)(x)=\gamma^{1}(s)(x)$ for $x \in\left(O^{i}\right)_{\varepsilon}^{2 \delta}, \gamma^{1, i}(s)(x)=0$ for $x \notin\left(O^{i}\right)_{\varepsilon}^{2 \delta}$. Note that $\left(A_{1}+\cdots+A_{n}-1\right)_{+} \geq \sum_{i=1}^{n}\left(A_{i}-1\right)_{+}$for $A_{1}, \cdots, A_{n} \geq 0$, and that $(p+1) / 2>1$. Then, we see that,

$$
\Gamma_{\varepsilon}\left(\gamma^{1}(s)\right) \geq \sum_{i=1}^{k} \Gamma_{\varepsilon}\left(\gamma^{1, i}(s)\right)=\sum_{i=1}^{k} \Gamma_{\varepsilon}^{i}\left(\gamma^{1, i}(s)\right)
$$

From Proposition 2 (ii) and since $\alpha \in(0, E-\tilde{E})$ we get that $\gamma^{1, i} \in \Phi_{\varepsilon}^{i}$, for all $i \in\{1, \cdots, k\}$. Thus by [9, Proposition 3.4], Proposition 3, (30) and (31) we deduce that

$$
\max _{s \in T} \Gamma_{\varepsilon}(\gamma(s)) \geq E+O(\varepsilon) .
$$

Since $\lim \sup _{\varepsilon \rightarrow 0} D_{\varepsilon} \leq E$ this contradicts (29) and completes the proof. 
Proposition 7. For sufficiently small fixed $\varepsilon>0, \Gamma_{\varepsilon}$ has a critical point $u_{\varepsilon} \in$ $X_{\varepsilon}^{d} \cap \Gamma_{\varepsilon}^{D \varepsilon}$.

Proof. Let $\left\{u_{n}\right\}_{n=1}^{\infty}$ be a Palais-Smale sequence as given by Proposition 6 corresponding to a fixed small $\varepsilon>0$. Since $\left\{u_{n}\right\}_{n=1}^{\infty}$ is bounded in $H_{\varepsilon}, u_{n} \rightarrow u$ weakly in $H_{\varepsilon}$, for some $u \in H_{\varepsilon}$. Then, it follows in a standard way that $u$ is a critical point of $\Gamma_{\varepsilon}$. Now we write $u_{n}=v_{n}+w_{n}$ with $v_{n} \in X_{\varepsilon}$ and $\left\|w_{n}\right\|_{\varepsilon} \leq d$. Since $X_{\varepsilon}$ is compact, there exists $v \in X_{\varepsilon}$ such $v_{n} \rightarrow v$ in $X_{\varepsilon}$, up to a subsequence, as $n \rightarrow \infty$. Moreover, for some $w \in H_{\varepsilon}, w_{n} \rightarrow w$ weakly, up to a subsequence, in $H_{\varepsilon}$, as $n \rightarrow \infty$. Thus, $u=v+w$ and

$$
\|u-v\|_{\varepsilon}=\|w\|_{\varepsilon} \leq \liminf _{n \rightarrow \infty}\left\|w_{n}\right\|_{\varepsilon} \leq d
$$

This proves that $u \in X_{\varepsilon}^{d}$.

To show that $\Gamma_{\varepsilon}(u) \leq D_{\varepsilon}$, it suffices to show that $\lim \sup _{n \rightarrow \infty} \Gamma_{\varepsilon}\left(u_{n}\right) \geq \Gamma_{\varepsilon}(u)$. In fact, writing $u_{n}=u+o_{n}$, we deduce that

$$
\begin{aligned}
\left\|o_{n}\right\|_{\varepsilon}=\left\|u_{n}-v-w\right\|_{\varepsilon} & \leq\left\|v_{n}-v\right\|_{\varepsilon}+\left\|w_{n}-w\right\|_{\varepsilon} \\
& \leq\left\|v_{n}-v\right\|_{\varepsilon}+\left\|w_{n}\right\|_{\varepsilon}+\|w\|_{\varepsilon} \\
& \leq 2 d+o(1)
\end{aligned}
$$

and

$$
\left\|u_{n}\right\|_{\varepsilon}^{2}=\|u\|_{\varepsilon}^{2}+\left\|o_{n}\right\|_{\varepsilon}^{2} .
$$

It is standard (see the proof of Proposition 2.31 in [10] for example) to show that

$$
\int_{\mathbf{R}^{N}} F\left(u_{n}\right) d x=\int_{\mathbf{R}^{N}} F(u) d x+\int_{\mathbf{R}^{N}} F\left(o_{n}\right) d x+o(1) .
$$

Thus we see that

$$
P_{\varepsilon}\left(u_{n}\right)=P_{\varepsilon}(u)+P_{\varepsilon}\left(o_{n}\right)+o(1) .
$$

Now

$$
P_{\varepsilon}\left(o_{n}\right)=\frac{1}{2}\left\|o_{n}\right\|_{\varepsilon}^{2}-\frac{1}{2} \int_{\mathbf{R}^{N}}\left(\tilde{V}_{\varepsilon}-V_{\varepsilon}\right) o_{n}^{2} d x-\int_{\mathbf{R}^{N}} F\left(o_{n}\right) d x .
$$

By (7), $\tilde{V}_{\varepsilon}-V_{\varepsilon}$ has a compact support. Thus from the weak convergence of $o_{n}$ in $H_{\varepsilon}$ it follows that $\int_{\mathbf{R}^{N}}\left(\tilde{V}_{\varepsilon}-V_{\varepsilon}\right) o_{n}^{2} d x \rightarrow 0$. Also from (f1),(f2), for some $C_{1}, C_{2}>0$

$$
\begin{aligned}
\int_{\mathbf{R}^{N}} F\left(o_{n}\right) d x & \leq \frac{\tilde{m}}{4} \int_{\mathbf{R}^{N}}\left(o_{n}\right)^{2} d x+C_{1} \int_{\mathbf{R}^{N}}\left(o_{n}\right)^{\frac{2 N}{N-2}} d x \\
& \leq \frac{1}{4}\left\|o_{n}\right\|_{\varepsilon}^{2}+C_{2}\left\|o_{n}\right\|_{\varepsilon}^{\frac{2 N}{N-2}} .
\end{aligned}
$$

Thus, for sufficiently large $n>0$ and small $d>0$, we have

$$
\frac{1}{2}\left\|o_{n}\right\|_{\varepsilon}^{2}-\int_{\mathbf{R}^{N}} F\left(o_{n}\right) d x \geq\left\|o_{n}\right\|_{\varepsilon}^{2}\left(\frac{1}{4}-C_{2}(3 d)^{\frac{4}{N-2}}\right)+o(1) \geq o(1) .
$$

It follows that $\lim \sup _{n \rightarrow \infty} \Gamma_{\varepsilon}\left(u_{n}\right) \geq \Gamma_{\varepsilon}(u)$ and this completes the proof.

We see from Proposition 7 that there exist $d>0$ and $\varepsilon_{0}>0$ such that, for $\varepsilon \in\left(0, \varepsilon_{0}\right), \Gamma_{\varepsilon}$ has a critical point $u_{\varepsilon} \in X_{\varepsilon}^{d} \cap \Gamma_{\varepsilon}^{D_{\varepsilon}}$. Since $u_{\varepsilon}$ satisfies

$$
\Delta u_{\varepsilon}-V_{\varepsilon} u_{\varepsilon}+f\left(u_{\varepsilon}\right)=(p+1)\left(\int \chi_{\varepsilon} u_{\varepsilon}^{2} d x-1\right)_{+}^{\frac{p-1}{2}} \chi_{\varepsilon} u_{\varepsilon} \text { in } \mathbf{R}^{N}
$$

and $f(t)=0$ for $t \leq 0$, we have that $u_{\varepsilon}>0$ in $\mathbf{R}^{N}$. Moreover, by elliptic estimates through Moser iteration scheme, we deduce that $\left\{\left\|u_{\varepsilon}\right\|_{L^{\infty}}\right\}_{\varepsilon}$ is bounded (see, for 
example, [4, Proposition 3.5] for such techniques). Now by Proposition 4, we see that

$$
\lim _{\varepsilon \rightarrow 0} \int_{\mathbf{R}^{N} \backslash M_{\varepsilon}^{\delta}}\left|\nabla u_{\varepsilon}\right|^{2}+\tilde{V}_{\varepsilon}\left(u_{\varepsilon}\right)^{2} d x=0 .
$$

Thus, by elliptic estimates (see [18]), we obtain that

$$
\lim _{\varepsilon \rightarrow 0}\left\|u_{\varepsilon}\right\|_{L^{\infty}\left(\mathbf{R}^{N} \backslash M_{\varepsilon}^{\delta} \cup Z_{\varepsilon}^{\delta}\right)}=0,
$$

and this gives the following decay estimate for $u_{\varepsilon}$ on $\mathbf{R}^{N} \backslash M_{\varepsilon}^{\delta} \cup Z_{\varepsilon}^{\delta}$.

Proposition 8. There exist some constants $C, c>0$ such that

$$
u_{\varepsilon}(x) \leq C \exp \left(-\operatorname{cdist}\left(x, M_{\varepsilon}^{\delta} \cup Z_{\varepsilon}^{\delta}\right)\right) .
$$

Proof. We note that $\inf \left\{V(x) \mid x \notin M_{\varepsilon}^{\delta} \cup Z_{\varepsilon}^{\delta}\right\}>0$. Then from (f1) and (33) we see that

$$
\lim _{\varepsilon \rightarrow 0}\left\|f\left(u_{\varepsilon}\right) / u_{\varepsilon}\right\|_{L^{\infty}\left(\mathbf{R}^{N} \backslash M_{\varepsilon}^{\delta} \cup Z_{\varepsilon}^{\delta}\right)}=0 .
$$

Thus, we obtain the decay estimate by applying a standard comparison principle (see [27]) to (32).

If $Z \neq \emptyset$ we need, in addition, the following estimate for $u_{\varepsilon}$ on $Z_{\varepsilon}^{2 \delta}$.

Proposition 9. There exist some constants $C, c>0$ such that

$$
\left\|u_{\varepsilon}\right\|_{L^{\infty}\left(Z_{\varepsilon}^{2 \delta}\right)} \leq C \exp (-c / \varepsilon) .
$$

Proof. Let $\left\{H_{\varepsilon}^{i}\right\}_{i \in I}$ be the connected components of $\operatorname{int}\left(Z_{\varepsilon}^{3 \delta}\right)$ for some index set $I$. Note that $Z \subset \cup_{i \in I} H_{\varepsilon}^{i}$ and $Z$ is compact. Thus the index set $I$ is finite. For each $i \in I$, let $\left(\phi^{i}, \lambda_{1}^{i}\right)$ be a pair of first positive eigenfunction and eigenvalue of $-\Delta$ on $H_{\varepsilon}^{i}$ with Dirichlet boundary condition. From now we fix an arbitrary $i \in I$. By (22), we see that for some constant $C>0$

$$
\left\|u_{\varepsilon}\right\|_{L^{\infty}\left(H_{\varepsilon}^{i}\right)} \leq C \varepsilon^{3 / \mu}
$$

(for such result see, for example, [18, Theorem 9.20]). Thus, from (f1) we have that for some $C>0$

$$
\left\|f\left(u_{\varepsilon}\right) / u_{\varepsilon}\right\|_{L^{\infty}\left(H_{\varepsilon}^{i}\right)} \leq C \varepsilon^{3} .
$$

Denote $\phi_{\varepsilon}^{i}(x)=\phi^{i}(\varepsilon x)$. Then, for sufficiently small $\varepsilon>0$, we deduce that for $x \in \operatorname{int}\left(H_{\varepsilon}^{i}\right)$,

$$
\Delta \phi_{\varepsilon}^{i}(x)-V_{\varepsilon}(x) \phi_{\varepsilon}^{i}(x)+\frac{f\left(u_{\varepsilon}(x)\right)}{u_{\varepsilon}(x)} \phi_{\varepsilon}^{i}(x) \leq\left(C \varepsilon^{3}-\lambda_{1} \varepsilon^{2}\right) \phi_{\varepsilon}^{i} \leq 0 .
$$

Now, since $\operatorname{dist}\left(\partial Z_{\varepsilon}^{2 \delta}, Z_{\varepsilon}^{\delta}\right)=\delta / \varepsilon$, we see from Proposition 8 that for some constants $C, c>0$,

$$
\left\|u_{\varepsilon}\right\|_{L^{\infty}\left(\partial Z_{\varepsilon}^{2 \delta}\right)} \leq C \exp (-c / \varepsilon)
$$

We normalize $\phi^{i}$ requiring that

$$
\inf \left\{\phi_{\varepsilon}^{i}(x) \mid x \in H_{\varepsilon}^{i} \cap \partial Z_{\varepsilon}^{2 \delta}\right\}=C \exp (-c / \varepsilon)
$$

for the same $C, c>0$ as in (36). Then, we see that for some $D>0$,

$$
\phi_{\varepsilon}^{i}(x) \leq D C \exp (-c / \varepsilon), x \in H_{\varepsilon}^{i} \cap Z_{\varepsilon}^{2 \delta} .
$$

Now we deduce, using (32), (35), (36), (37) and [29, B.6 Theorem] that for each $i \in I, u_{\varepsilon} \leq \phi_{\varepsilon}^{i}$ on $H_{\varepsilon}^{i} \cap Z_{\varepsilon}^{2 \delta}$. Therefore $u_{\varepsilon}(x) \leq C \exp (-c / \varepsilon)$ on $Z_{\varepsilon}^{2 \delta}$ for some $C, c>0$ and this completes the proof. 
Now we can complete the proof of Theorem 1. From Propositions 8 and 9 we see that $Q_{\varepsilon}\left(u_{\varepsilon}\right)=0$ for sufficiently small $\varepsilon>0$ and then (32) shows that $u_{\varepsilon}$ satisfies (6). Now the properties (i) and (ii) of $v_{\varepsilon}(x) \equiv u_{\varepsilon}(x / \varepsilon)$ in Theorem 1 follow directly from Propositions 1 and 4 .

Acknowledgements. This work of the first author was supported by grant No. R01-2004-000-10055-0 from the Basic Research Program of the Korea Science \& Engineering Foundation. This work was initiated during a visit of the second author to the mathematical department of POSTECH in April 2005. He would like to express his gratitude for this invitation and support.

\section{REFERENCES}

[1] A. Ambrosetti, M. Badiale and S. Cingolani, Semiclassical states of nonlinear Schrödinger equations, Arch. Ration. Mech. Anal., 140 (1997), 285-300.

[2] A. Ambrosetti, A. Malchiodi and S. Secchi, Multiplicity results for some nonlinear Schrödinger equations with potentials, Arch. Ration. Mech. Anal., 159 (2001), 253-271.

[3] H. Berestycki and P.L. Lions, Nonlinear scalar field equations I, Arch. Ration. Mech. Anal., 82 (1983), 313-346.

[4] J. Byeon, Existence of large positive solutions of some nonlinear elliptic equations on singularly perturbed domains, Comm. in P.D.E., 22 (1997), 1731-1769.

[5] J. Byeon and L. Jeanjean, Standing waves for nonlinear Schrödinger equations with a general nonlinearity, Arch. Ration. Mech. Anal., to appear.

[6] J. Byeon and Y. Oshita, Existence of multi-bump standing waves with a critical frequency for nonlinear Schrödinger equations, Comm. in P.D.E., 29 (2004), 1877-1904.

[7] J. Byeon and Z.-Q. Wang, Standing waves with a critical frequency for nonlinear Schrödinger equations, Arch. Ration. Mech. Anal., 165 (2002), 295-316.

[8] J. Byeon and Z.-Q. Wang, Standing waves with a critical frequency for nonlinear Schrödinger equations II, Calculus of Variations and PDE, 18 (2003), 207-219.

[9] V. Coti Zelati and P.H. Rabinowitz, Homoclinic orbits for second order Hamiltonian systems possessing superquadratic potentials, J. Amer. Math. Soc., 4 (1991), 693-727.

[10] V. Coti-Zelati and P.H. Rabinowitz, Homoclinic type solutions for a semilinear elliptic PDE on $\mathbf{R}^{N}$, Comm. on Pure Appl. Math., 45 (1992), 1217-1269.

[11] E.N. Dancer, K.Y. Lam and S. Yan, The effect of the graph topology on the existence of multipeak solutions for nonlinear Schrödinger equations, Abstr. Appl. Anal., 3 (1998), 293318.

[12] E.N. Dancer and S. Yan, On the existence of multipeak solutions for nonlinear field equations on $\mathbf{R}^{N}$, Discrete Contin. Dynam. Systems, 6, (2000), 39-50.

[13] M. Del Pino and P.L. Felmer, Local mountain passes for semilinear elliptic problems in unbounded domains, Calculus of Variations and PDE, 4, (1996), 121-137.

[14] M. Del Pino and P.L. Felmer, Semi-classical states for nonlinear Schrödinger equations, J. Functional Analysis, 49, (1997), 245-265.

[15] M. Del Pino and P.L. Felmer, Multi-peak bound states for nonlinear Schrödinger equations, Ann. Inst. Henri Poincaré, 15, (1998), 127-149.

[16] M. Del Pino and P.L. Felmer, Semi-classical states for nonlinear Schrödinger equations: a variational reduction method, Math. Ann., 324, 1, (2002), 1-32.

[17] A. Floer and A. Weinstein, Nonspreading wave packets for the cubic Schrödinger equations with a bounded potential, J. Functional Analysis, 69, (1986), 397-408.

[18] D. Gilbarg and N. S. Trudinger, Elliptic Partial Differential Equations of Second Order; second edition, Grundlehren 224, Springer, Berlin, Heidelberg, New York and Tokyo, (1983).

[19] C. Gui, Existence of multi-bump solutions for nonlinear Schrödinger equations via variational method, Comm. in P.D.E., 21, (1996), 787-820.

[20] L. Jeanjean and K. Tanaka, A remark on least energy solutions in $\mathbf{R}^{N}$, Proc. Amer. Math. Soc. 131, (2003), 2399-2408.

[21] L. Jeanjean and K. Tanaka, Singularly perturbed elliptic problems with superlinear or asymptotically linear nonlinearities, Calculus of Variations and PDE, 21, (2004), 287-318.

[22] X. Kang and J. Wei, On interacting bumps of semi-classical states of nonlinear Schrödinger equations, Adv. Differential Equations, 5, (2000), 899-928. 
[23] Y.Y. Li, On a singularly perturbed elliptic equation, Adv. Differential Equations, 2, (1997), 955-980.

[24] P.L. Lions, The concentration-compactness principle in the calculus of variations. The locally compact case, part II , Ann. Inst. Henri Poincaré, 1, (1984), 223-283.

[25] Y.G. Oh, Existence of semiclassical bound states of nonlinear Schrödinger equations with potentials of the class $(V)_{a}$, Comm. P.D.E., 13, (1988), 1499-1519.

[26] Y.G. Oh, On positive multi-lump bound states of nonlinear Schrödinger equations under multiple well potential, Comm. Math. Phys., 131, (1990), 223-253.

[27] M. H. Protter and H. F. Weinberger, Maximum Principles in Differential Equations, SpringerVerlag, New York, Berlin, Heidelberg and Tokyo, 1984.

[28] P.H. Rabinowitz, On a class of nonlinear Schrödinger equations, Z. Angew. Math. Phys., 43 (1992), 270-291.

[29] M. Struwe, Variational Methods; Application to Nonlinear Partial Differential Equations and Hamiltonian Systems, Springer-Verlag, 1990.

[30] X. Wang, On concentration of positive bound states of nonlinear Schrödinger equations, Comm. Math. Phys., 153, (1993), 229-244.

E-mail address: jbyeon@postech.ac.kr; louis.jeanjean@univ-fcomte.fr 